\title{
Automated digital reporting of clinical laboratory information to national public health surveillance systems, results of a EU/EEA survey, 2018
}

Katrin Claire Leitmeyer ${ }^{1}$, Laura Espinosa ${ }^{1}$, Eeva Kaarina Broberg ${ }^{1}$, Marc Jean Struelens ${ }^{1}$, the ECDC National Focal Points laboratory e-reporting survey group members ${ }^{2}$

1. European Centre for Disease Prevention and Control (ECDC), Stockholm, Sweden

2. The ECDC National Focal Points laboratory e-reporting survey group members are listed at the end of the article

Correspondence: Katrin Leitmeye (Katrin.Leitmeyer@ecdc.europa.eu)

\section{Investigator group:}

ECDC National Focal Points laboratory e-reporting survey group: Franz Allerberger, Yves Dupont, Steven Van Gucht, Sophie Quoilin, Iva Christova, Blazenka Hunjak, Christos Karagiannis, Pavla Křížová, Jitka Cástková, Eva Møller Nielsen, Jonas Kähler, Rita Peetso, Saara Salmenlinna, Teemu Möttönen, Bruno Coignard, Michaela Diercke, Alkiviadis Vatopoulos, Ákos Tóth, Karl Gustaf Kristinsson, Eleanor McNamara, Annalisa Pantosi, Violeta Mavcutko, Algirdas Griškevičius, Joël Mossong, Christopher Barbara, Titia Kortbeek, Dominique Caugant, Ulf Dahle, Line Vold, Anna Skoczyńska, Jorge Machado, Gabriel lonescu, Lucia Madarova, Mária Avdičová, Metka Paragi, Julio Moreno Vazquez, Karin Tegmark Wisell, Maria Zambon

Citation style for this article:

Leitmeyer Katrin Claire, Espinosa Laura, Broberg Eeva Kaarina, Struelens Marc Jean, the ECDC National Focal Points laboratory e-reporting survey group members Automated digital reporting of clinical laboratory information to national public health surveillance systems, results of a EU/EEA survey, 2018 . Euro Surveill.

2020;25(39):pii=1900591. https://doi.org/10.2807/1560-7917.ES.2020.25·39.1900591

Background: Timely reporting of microbiology test results is essential for infection management. Automated, machine-to-machine $\left(\mathrm{M}_{2} \mathrm{M}\right)$ reporting of diagnostic and antimicrobial resistance (AMR) data from laboratory information management systems (LIMS) to public health agencies improves timeliness and completeness of communicable disease surveillance. Aim: We surveyed microbiology data reporting practices for national surveillance of EU-notifiable diseases in European Union/European Economic Area (EU/EEA) countries in 2018. Methods: European Centre for Disease Prevention and Control (ECDC) National Microbiology and Surveillance Focal Points completed a questionnaire on the modalities and scope of clinical microbiology laboratory data reporting. Results: Complete data were provided for all 30 EU/EEA countries. Clinical laboratories used a LIMS in 28 countries. LIMS data on EU-notifiable diseases and AMR were $\mathrm{M}_{2} \mathrm{M}$-reported to the national level in 14 and nine countries, respectively. In the 14 countries, associated demographic data reported allowed the de-duplication of patient reports. In 13 countries, M2M-reported data were used for cluster detection at the national level. M2M laboratory data reporting had been validated against conventional surveillance methods in six countries, and replaced those in five. Barriers to M2M reporting included lack of information technology support and financial incentives. Conclusion: M2M-reported laboratory data were used for national public health surveillance and alert purposes in nearly half of the EU/EEA countries in 2018. Reported data on infectious diseases and AMR varied in extent and disease coverage across countries and laboratories. Improving automated laboratory-based surveillance will depend on financial and regulatory incentives, and harmonisation of health information and communication systems.

\section{Introduction}

Electronic laboratory-based data reporting has been shown to be an effective and efficient method to automate and improve the timeliness and completeness of communicable diseases and antimicrobial resistance (AMR) surveillance at the healthcare institution level, as well as the national public health level [1-10].

The routine use of digital laboratory data for surveillance purposes has been reported from European countries such as Denmark [11,12] and France [6,13] However, the extent to which such automated laboratory data reporting methods from clinical diagnostic laboratories to national surveillance systems are used has not been mapped systematically in the European Union (EU) and the European Economic Area (EEA).

As a part of the monitoring of EU/EEA national laboratory capacities for public health surveillance and control of infectious diseases, 17 of 30 EU/EEA countries reported, that in 2016, their surveillance networks of clinical laboratories reported microbiology data for at least one disease by machine-to-machine (M2M) upload to a central national public health database [14]. The European Centre for Disease Prevention and Control (ECDC) Public Health Microbiology Strategy [15] foresees that ECDC will, in synergy with relevant European Commission-supported eHealth initiatives, undertake projects to identify and disseminate solutions for automated $\left(\mathrm{M}_{2} \mathrm{M}\right)$ transfer of microbiology data between laboratory and surveillance information systems from the local to national and EU/EEA 


\section{Box}

EU priority indicator antimicrobial-resistant pathogens [19] and antiviral-resistant pathogens in humans under EU/EEA surveillance

- Meticillin-resistant Staphylococcus aureus

- 3rd-generation cephalosporin-resistant Escherichia coli

- Klebsiella pneumoniae resistant to aminoglycosides, fluoroquinolones and 3 rd-generation cephalosporins

- Streptococcus pneumoniae resistant to penicillin and macrolides

- Klebsiella pneumoniae resistant to carbapenems

- HIV resistant to anti-retroviral agents and influenza virus resistant to neuraminidase inhibitors

EU/EEA: European Union/European Economic Area.

surveillance levels. The ECDC strategy proposes as target that in 2022 , at least $90 \%$ of EU/EEA countries will be using automated electronic notification of clinical laboratory data to national surveillance programmes.

The primary objective of this survey was to investigate the modalities and scope of clinical microbiology laboratory data reporting for the national surveillance of EU-notifiable diseases and AMR across Europe. It also assessed the public health use of this information for rapid threat detection and timely response.

\section{Methods}

\section{Survey tool}

The questionnaire administered in the EU survey tool (https://ec.europa.eu/) was developed by ECDC in consultation with the National Microbiology Focal Points (NMFPs) and National Surveillance Focal Points (NSFPs) who are the EU/EEA countries' designated experts for advice on specific public health functions to ECDC as outlined in the Terms of reference, Annex 3 [16]. NMFPs and NSFPs provided written comments to the first draft of the questionnaire, and carried out a pilot study to check for clarity and usability before launching the survey. Using a Delphi-like approach, two face-to-face discussions were conducted between the study coordinators and survey respondents: the first in October 2018 to revise the draft questionnaire, and the second in June 2019 to critically review and clarify the correct interpretations of terms and definitions used to describe the national reporting systems in order to enhance inter-observer consistency. Furthermore, individual teleconferences were conducted with NMFPs between the two discussions. The final questionnaire included 21 questions supplemented with a glossary of terms.

The questionnaire asked the NMFPs and NSFPs to describe the following items at the national level:

- Use of laboratory information management systems (LIMS) in clinical diagnostic laboratories for reporting test results to clinicians;
- Modes of diagnostic data reporting from clinical diagnostic laboratories to national surveillance databases for the 56 EU-notifiable diseases [17], and for reporting susceptibility data on EU priority indicator antimicrobial-resistant pathogens $[18,19]$ and antiviral-resistant viral pathogens under EU/EEA surveillance (Box);

- Proportion and type of clinical diagnostic laboratories that report data by automated $\mathrm{M}_{2} \mathrm{M}$ upload from their LIMS to national surveillance system databases (public laboratories, for profit commercial laboratories, non-for profit private laboratories, academic hospital laboratories, national public health microbiology reference laboratories);

- Reasons for non-automated laboratory data reporting to surveillance databases (lack of legal basis/ obligation, lack of financial incentive to cover extra cost, lack of information technology (IT) support for data reporting, data protection issues, lack of relevance for surveillance);

- Use of daily or weekly transmitted laboratory data for continuous/frequent cluster event detection and early warning at the national public health level;

- Type and scope of laboratory test data and of patient demographic, clinical and epidemiological data reported on automated basis from LIMS to national surveillance databases;

- Possibility of laboratory data linkage to epidemiological data collected from other sources (e.g. medical case notification);

- Data checks and quality controls (e.g. de-duplication for multiple repeat positive samples per patient);

- Previous epidemiological validation study of the laboratory-based automated electronic surveillance method against conventional epidemiological surveillance methods (e.g. conventional case notification-based or questionnaire-based data collection methods for surveillance of a particular disease);

- Replacement of conventional/case-based reporting epidemiological surveillance protocols by automated M2M laboratory-based reporting systems;

- Use of healthcare vocabulary/terminology standards (e.g. Logical Observation Identifiers Names and Codes (LOINC)-controlled terminology, International Statistical Classification of Diseases and Related Health Problems, 10th revision (ICD-10) medical classification list in LIMS-generated data format and the Systematic Nomenclature of Medicine Clinical Terms (SNOMED-CT)); and 


\section{FIGURE 1}

Clinical laboratories reporting data by automated machine-to-machine communication to national surveillance databases, 30 EU/EEA countries, December 2018
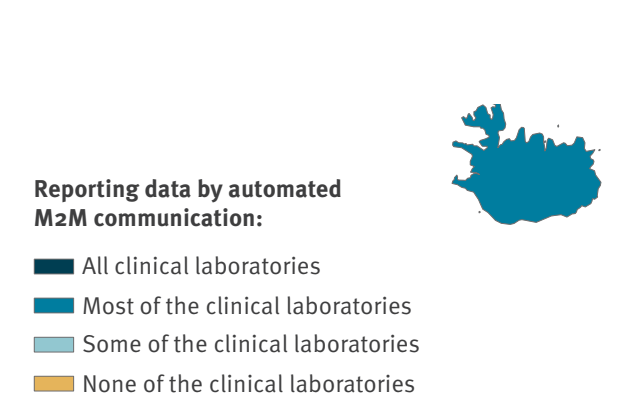

\footnotetext{
Countries not visible:

$\square$ Luxembourg

$\square$ Malta
}

Map produced on: 27 May 2020

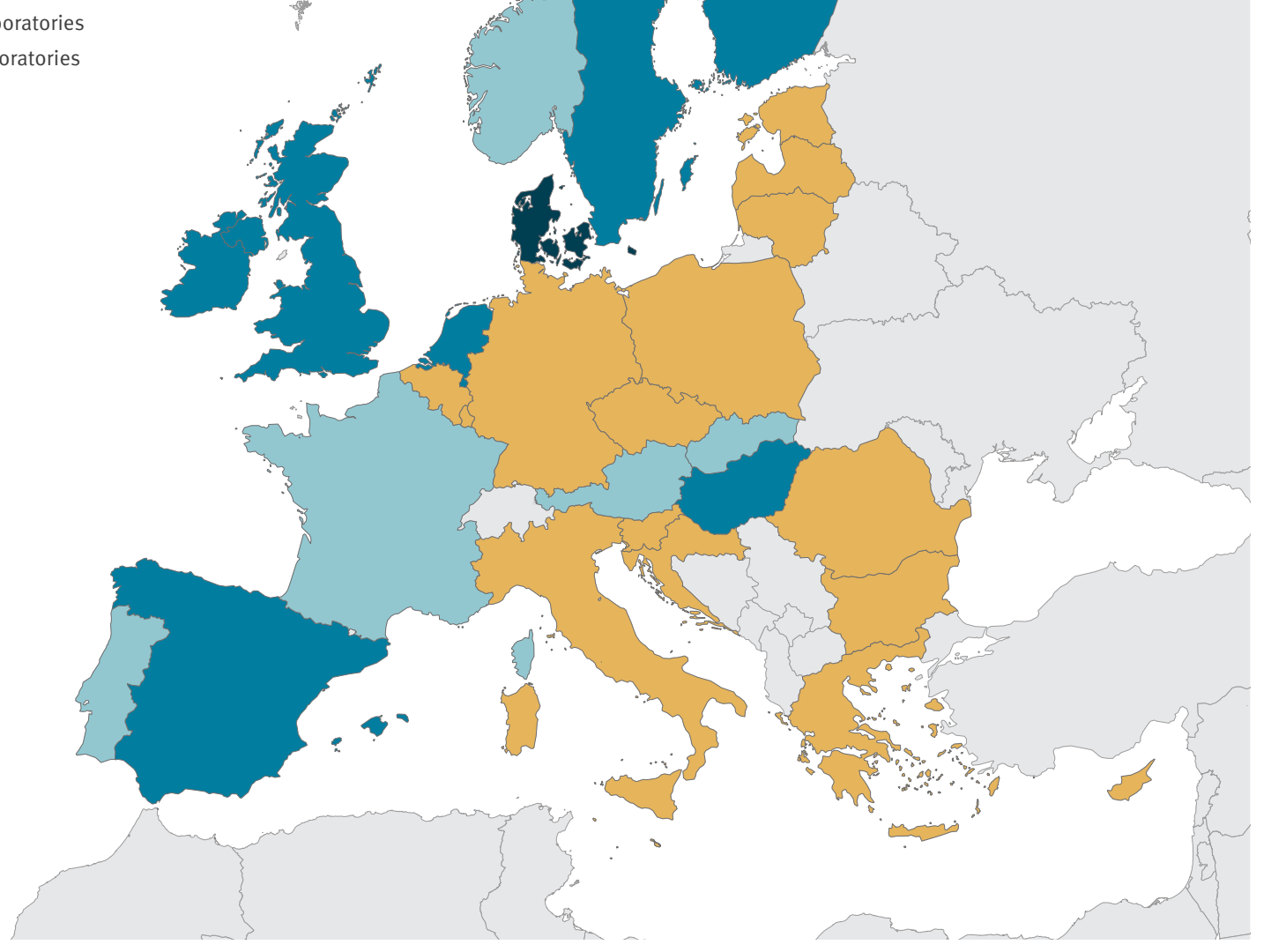

EU/EEA: European Union/European Economic Area; M2M: machine-to-machine.

The question in the questionnaire was categorical, with response options 'all', 'most', 'some' or 'none'.

- Plans, if any, to use automated digital laboratory information reporting systems in the near future for public health purposes.

\section{Definitions used}

Laboratory information management system

A LIMS is a software system developed to support laboratory operations including results communication. 'This software system can track specimens and workflows, aggregate data for research or business intelligence purposes, and ensure laboratory operations are compliant with various standards and regulations' [20].

Machine-to-machine communication

'Any technology that enables networked devices to exchange information and perform actions without the manual assistance of humans' [21]. This automated communication follows an application programming interface (API), using a set of clearly defined methods of electronic communication among various IT components.

Automated machine-to-machine laboratory data reporting

For this study, this is defined as the direct, automated $\mathrm{M}_{2} \mathrm{M}$ upload of reportable disease laboratory data from clinical LIMS to the national communicable disease surveillance system. This definition is similar to that of Electronic Laboratory Reporting (ELR) used in the United States (US) [22]. It should be noted that the automated transmission may need prior verification/ signed authorisation by the clinical laboratory director, in particular where the transferred data relate to mandatory disease notification by a medical doctor. 


\section{FIGURE 2}

Countries reporting EU-notifiable disease data from clinical diagnostic laboratories to national surveillance databases, by disease and reporting method ${ }^{\mathrm{a}}, 30$ EU/EEA countries, December 2018

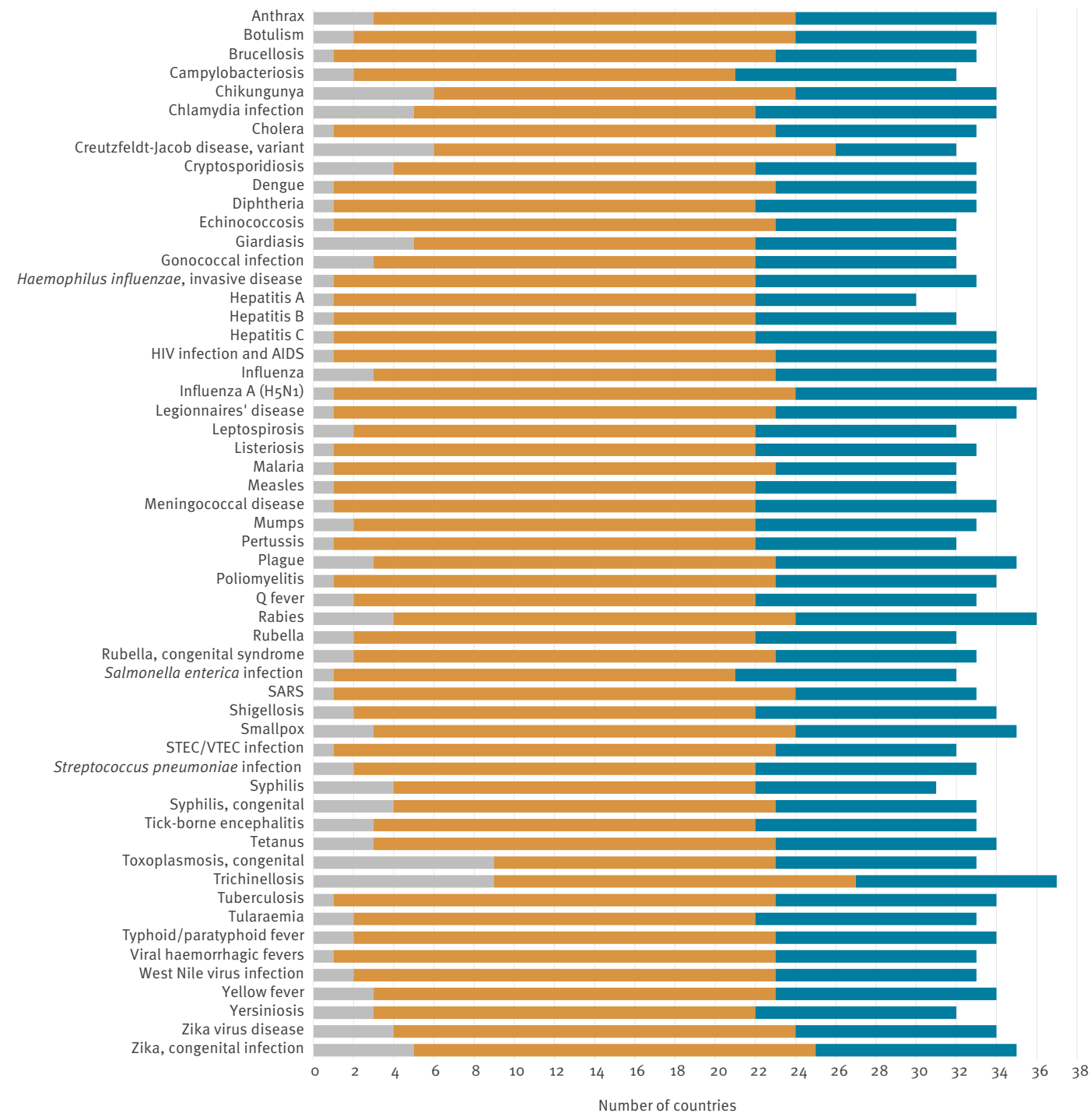

Type of reporting to national surveillance databases:

No reporting Manual reporting Automated reporting

EU/EEA: European Union/European Economic Area; SARS: severe acute respiratory syndrome; STEC/VTEC: Shiga-toxin/verocytotoxinproducing Escherichia coli.

${ }^{\text {a }}$ Multiple answers possible.

Manual laboratory data reporting

As defined for this study, this includes any other mode of laboratory data reporting, including paper-based reporting by postal mail, facsimile or email; manual reporting by entering data into web-based questionnaire forms and manual file extraction; and upload via the Internet to a central webpage or online database.

\section{Data collection, validation and analysis}

The survey was distributed by email on 25 October 2018 to the NMFPs of 28 EU countries and two EEA countries (Liechtenstein was not included), keeping in copy the National Coordinators of national public health authorities to ensure the best informed respondent in each EU/EEA country was selected to complete the survey. The survey collected information on the 2018 capabilities and capacities of the countries, and was open until 10 December 2018. A report with the preliminary results of the survey was shared with participants on 18 December for their review, and the survey tool was reopened until July 2019 after the results of a preliminary analysis for national data verification and completion of reporting gaps. All questionnaires containing inconsistent or incomplete answers were discussed bilaterally between the survey coordinator and 


\section{FIGURE 3}

Automated reporting of clinical laboratory data (diagnostic and antimicrobial susceptibility testing data a $^{\text {) to national }}$ surveillance databases, 14 EU/EEA countries with automated laboratory-based surveillance capability, December 2018

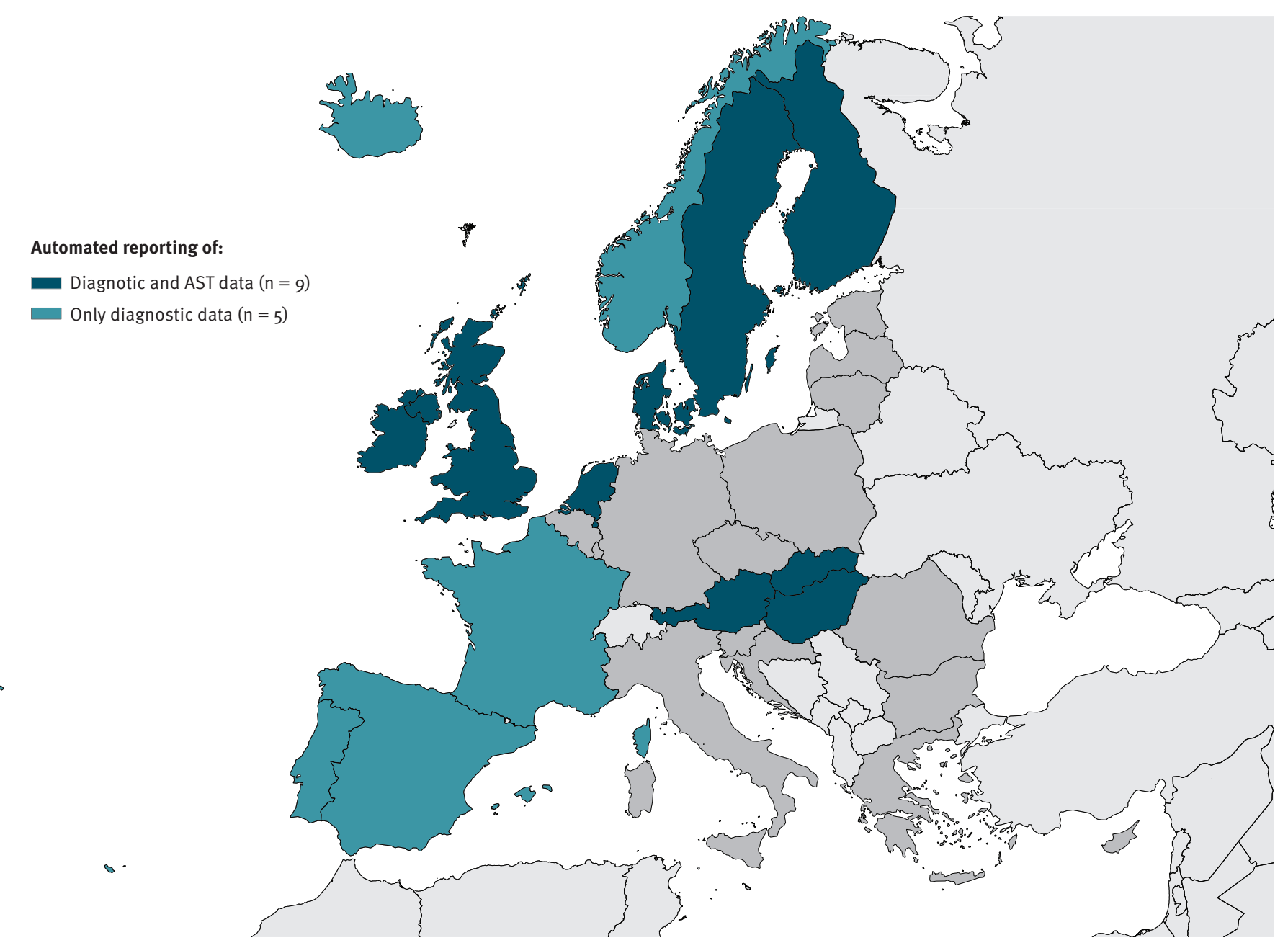

AST: antibiotic susceptibility testing; EU/EEA: European Union/European Economic Area.

a Diagnostic and susceptibility data refer to all or some EU priority indicator antimicrobial-resistant pathogens.

the respondents. Data were thereby completed and validated with the participating experts in the country before inclusion in the final analysis. Data completeness was calculated as a percentage of reported data for each question. Data are presented using descriptive summary statistics.

\section{Ethical statement}

For this survey, we did not seek any ethical review as no personal data were collected. The release of the manuscript including anonymised interview data from the national experts has been approved by all authors.

\section{Results}

Response rate and data completeness

Each of the 30 EU/EEA countries provided a complete response the survey, i.e. all countries responded to all 21 questions. There were only four 'I do not know' replies, by one country each to a different question.
Use of laboratory information management systems for data reporting from clinical diagnostic laboratories to clinicians In 2018, a LIMS was used in 28 of the countries by all $(n=9$ countries), most $(n=14$ countries) or some $(n=5$ countries) clinical diagnostic laboratories to manage and report laboratory test results to clinicians. In Bulgaria and Latvia, clinical diagnostic laboratories did not use any LIMS.

\section{Mode of laboratory data reporting from clinical laboratories to national surveillance databases}

In 16 countries, clinical diagnostic laboratories only reported data manually to national surveillance databases, while in 14 countries, all ( $n=1$ country), most ( $n=8$ countries) or some $(n=5$ countries) clinical diagnostic laboratories reported digital data automatically M2M from their LIMS to national databases (Figure 1). In Denmark, all clinical laboratories used automated $\mathrm{M}_{2} \mathrm{M}$ reporting to the national surveillance databases 


\section{FIGURE 4}

Modes clinical diagnostic laboratories use to report EU priority indicator antimicrobial-resistant pathogens ${ }^{\mathrm{a}}$ to national surveillance databases, 14 EU/EEA countries with automated laboratory-based surveillance capability, December 2018

Streptococcus pneumoniae, penicillin-resistant

Staphylococcus aureus, meticillin-resistant (MRSA)

Klebsiella pneumoniae, carbapenem-resistant

Streptococcus pneumoniae, macrolide-resistant

Mycobacterium tuberculosis, first-line-resistant

Escherichia coli, $3 \mathrm{GCR}$

Klebsiella pneumoniae, aminoglycoside-, fluoroquinoloneresistant and $3 \mathrm{GCR}$

Clostridioides difficile, first-line-resistant

HIV virus, anti-retroviral-resistant

Influenza virus, neuraminidase inhibitor-resistant

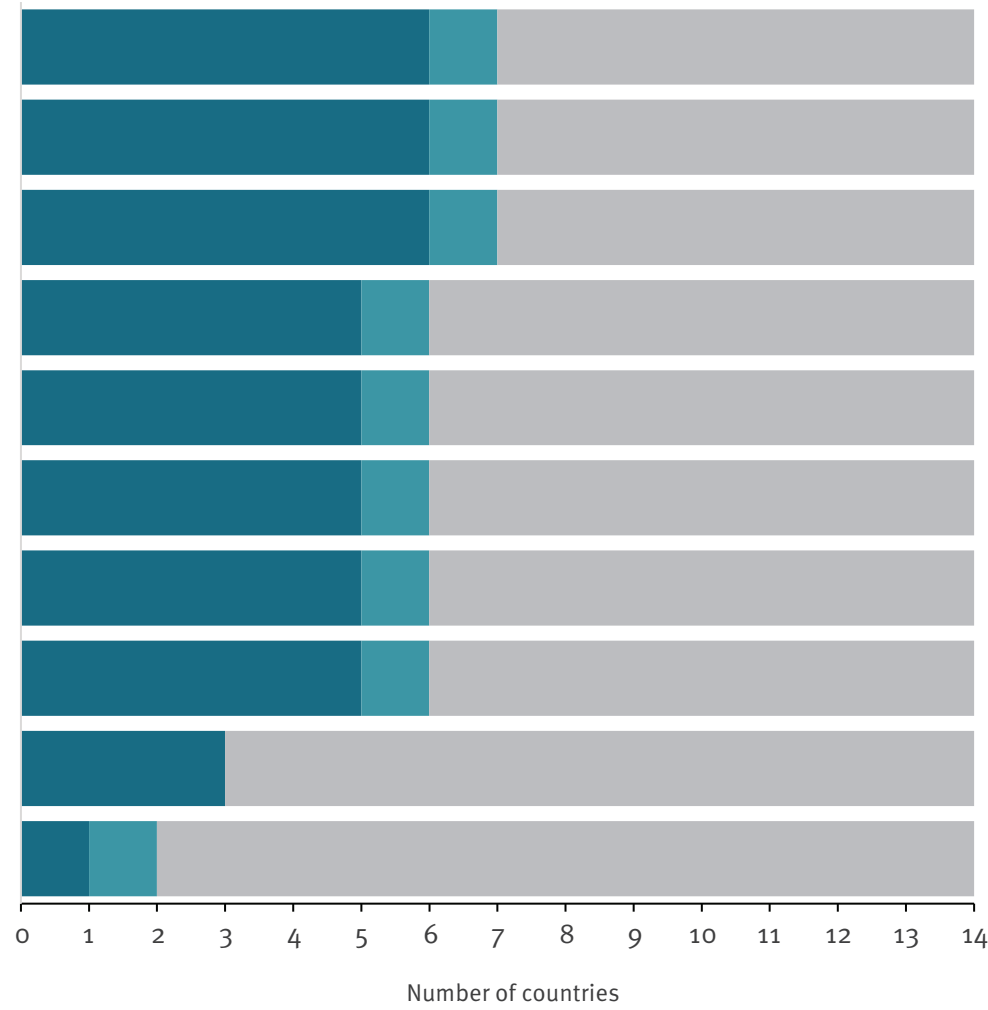

Type of reporting:

Automated $\quad$ Automated and other

EU/EEA: European Union/European Economic Area; 3GCR: third generation cephalosporin-resistant.

a See [19].

without any manual intervention. In another 13 countries, either most diagnostic laboratories (Finland, Hungary, Iceland, Ireland, the Netherlands, Spain, Sweden and the UK) or some diagnostic laboratories (Austria, France, Norway, Portugal and Slovakia) reported data by automated $\mathrm{M}_{2} \mathrm{M}$ communication to national databases. (Figure 1). Diverse automated reporting systems were used by laboratories in six countries whereas a single, generic reporting system for all diseases was available in eight countries. The majority of the countries $(n=25)$ had plans to start using or further expand their automated laboratory information reporting systems in the near future for public health purposes.

\section{Type of laboratories reporting by automated machine-to-machine communication to national databases}

Among the 14 countries with automated M2M laboratory data reporting to national surveillance databases, diagnostic laboratories from the public sector reported on an automated basis to national databases in 12 countries, commercial diagnostic laboratories in 10 countries and academic hospital laboratories in eight countries. National reference laboratories also reported on an automated basis to national databases in 10 countries.

\section{Laboratory data reporting for EU-notifiable diseases}

Figure 2 shows the proportion of EU/EEA countries where EU-notifiable disease related laboratory data were reported to national surveillance databases in 2018 by disease and reporting method. Laboratory data on some parasitic diseases, e.g. trichinellosis and congenital toxoplasmosis, and/or rare diseases, e.g. variant Creutzfeldt-Jacob disease (vCJD), chikungunya, were least frequently reported by laboratories using either manual or automated methods. Overall 23 of 56 notifiable diseases were reported from the laboratory to a national database in all but one country. Among the diseases reported to national surveillance databases, the number of countries reporting laboratory data automated was six for VCJD and ranged from nine to 12 for the other notifiable diseases. 
Type of data reported automatically to national surveillance databases, 14 EU/EEA countries with automated laboratorybased surveillance capability, December 2018

\begin{tabular}{|c|c|}
\hline Data type & $\begin{array}{l}\text { Number of countries reporting on automated basis } \\
\qquad(\mathrm{n}=14)\end{array}$ \\
\hline \multicolumn{2}{|l|}{ Laboratory test data } \\
\hline Type of clinical specimen tested & 13 \\
\hline Type of diagnostic test performed & 10 \\
\hline Number of clinical specimens tested & 6 \\
\hline Number of diagnostic tests performed & 3 \\
\hline Positive diagnostic test results & 12 \\
\hline Quantitative diagnostic test results (e.g. serology results, NAT results) & 6 \\
\hline Negative diagnostic test results & 4 \\
\hline \multicolumn{2}{|l|}{ Patient demographic data } \\
\hline Age & 14 \\
\hline Sex & 14 \\
\hline Place of residence & 13 \\
\hline \multicolumn{2}{|l|}{ Clinical and epidemiological data } \\
\hline Clinical diagnosis & 8 \\
\hline Underlying disease & 4 \\
\hline Treatments & 3 \\
\hline Vaccination status & 7 \\
\hline Date of onset of disease & 9 \\
\hline Community/healthcare-associated disease & 6 \\
\hline Travel history & 9 \\
\hline
\end{tabular}

EU/EEA: European Union/European Economic Area; NAT: nucleic acid amplification testing.

Reporting on indicator antimicrobial-resistant pathogens and antibiotic susceptibility data Data on some or all the EU priority indicator antimicrobial-resistant pathogens were reported from clinical laboratories by automated $\mathrm{M}_{2} \mathrm{M}$ communication to the national surveillance level in nine of 14 countries with automated laboratory-based surveillance capability (Figure 3).

Antibiotic susceptibility testing (AST) results were reported in those nine countries by automated $\mathrm{M}_{2} \mathrm{M}$ communication in a qualitative format (categorised as 'resistant', 'intermediate', 'susceptible') according to European Committee on Antimicrobial Susceptibility Testing (EUCAST) 2018 version 8.0 definitions and clinical breakpoints [23]. In addition, five of these countries automatically also reported AST data in quantitative formats, like the disk diffusion zone size or the minimum inhibitory concentration (MIC). Positive results for detection and identification of specific AMR mechanism/determinants, e.g. extended-spectrum beta-lactamases, were further reported automatically in seven of these countries (Figure 4). For bacterial pathogens, approximately half of the 14 countries reported AMR data by automated $\mathrm{M}_{2} \mathrm{M}$ communication, while resistance to viral pathogens such as HIV and influenza virus was predominantly reported manually.

\section{Data use for cluster detection and early} warning at national level

In all but one country with automated M2M laboratory data reporting, the daily or weekly transmitted data were used at the national level for continuous or frequent cluster event detection analysis and early warning for public health purpose. Five countries analysed these data for all the pathogens reported to the national system, while the remaining eight countries did this only for selected diseases. The latter included respiratory diseases (influenza, Mycoplasma pneumoniae infection, respiratory syncytial virus infection), food-borne diseases (salmonellosis, campylobacteriosis, shigellosis, listeriosis), sexually transmitted diseases (syphilis, gonorrhoea, chlamydia infection, viral hepatitis, HIV infection), multidrug-resistant pathogens (multidrug-resistant tuberculosis, meticillinresistant Staphylococcus aureus (MRSA)) and arbovirus infections/viral haemorrhagic fevers.

Type of data reported by automated machineto-machine communication

The type of data reported automatically by $\mathrm{M}_{2} \mathrm{M}$ from clinical laboratories' LIMS to national surveillance systems in 14 EU/EEA countries is described in the Table. The type of clinical specimens tested was more systematically reported than the type of diagnostic tests performed. Positive test results were more frequently reported than negative test results. Denominator data on the total number of diagnostic tests performed and 
the number of clinical specimen tested were reported by laboratories in less than half of these countries. Basic patient demographic data (age and sex) were reported in all countries whereas data on patient place of residence were also reported in most countries. Patient clinical and epidemiological data were reported in approximately half of these 14 countries on the vaccination status (immunisation history), and the healthcare or community association of the diagnosed infection. Meanwhile, nine of 14 countries reported data from LIMS on patient history of recent travel outside their country of residence.

\section{Semantic coding and data cross-linkage}

Regarding the interoperability formats of messages from $\mathrm{M}_{2} \mathrm{M}$ laboratory reporting, standard healthcare vocabulary or controlled coding terminology was used in seven countries. The coding standards include ICD10 in three countries and SNOMED-CT in one. The microbiological diagnostic test codes comparable to the LOINC was used in two countries. Two countries did not specify the vocabulary used.

In seven of the 14 countries using automated $M_{2} M$ reporting, the reported laboratory data were automatically linked to case-based notified epidemiological data. In six countries, the laboratory data were also automatically linked to other databases. Personal data protection issues and information governance controls were mentioned as main obstacles in the countries not cross-linking the data.

\section{Data quality and epidemiological validation} of electronically reported laboratory data for surveillance

National surveillance systems allowed for the de-duplication of positive samples per patient in all 14 countries reporting data $M_{2} M$. In six countries, the accuracy of the laboratory-based automated electronic surveillance methods was reported to have been epidemiologically validated against standard epidemiological surveillance methods such as manual case notification or questionnaire-based data collection methods for disease surveillance. In five of these 14 countries, automated $\mathrm{M}_{2} \mathrm{M}$ laboratory reporting systems had replaced some of the conventional epidemiological surveillance protocols using case-based reporting.

\section{Obstacles to automated laboratory reporting} The two most frequent reasons why clinical laboratories were not automatically reporting data to national surveillance databases by $\mathrm{M} 2 \mathrm{M}$ communication were a lack of technical IT support $(n=17$ countries) and a lack of financial compensation for data reporting $(n=15$ countries). In seven countries, there was no legal basis/ obligation for automated laboratory data reporting and in four countries, personal data protection was cited as a major obstacle for automated reporting. Further reasons mentioned included the non-relevance of such data for surveillance, data ownership, the diversity of LIMS within a country, and lack of standardisation for coding dictionaries, computer systems and computer security systems.

\section{Discussion}

This report on current EU/EEA practices and obstacles at the national level offers a basis for surveillance system improvement and efficiency gains through automated laboratory data reporting. It further provides perspective for future automated EU-wide laboratory based surveillance.

Timely and complete reporting of diagnostic microbiology test results to different stakeholders is essential for effective medical and public health infection management.

Within the EU/EEA, the exact number of clinical microbiology laboratories by country is not known. According to a membership survey by the European Society of Clinical Microbiology and Infectious Diseases (ESCMID), the number of microbiological service providers in Europe ranged widely from four to 69 laboratories per 10 million inhabitants in 2011 [24].

ECDC published an overview by notifiable disease of the national surveillance systems operating in 2016, indicating differences and commonalities across the EU/EEA [25]. However, individual countries' use of standardised vocabulary and data constraints were not described. The present survey is the first to map the status of automation of microbiology data reporting from clinical LIMS to the national public health level in the EU/EEA.

In 2018, about half of the EU/EEA countries had electronic reporting systems in place for automated surveillance of notifiable infectious diseases by $M_{2} M$ data transfer from clinical laboratories to the national surveillance databases. This compares with a progress report from US Centers for Disease Control and Prevention (CDC) indicating that $31 \%$ of the 10,600 reporting clinical laboratories from 55 of 57 jurisdictions in the US were reporting data electronically to the CDC in 2014 [26]. This process had been supported with national funding since 2010 through the Epidemiology and Laboratory Capacity for Infectious Diseases cooperative agreement. The proportion of electronic laboratory reporting of surveillance data across the EU/EEA countries varied by disease, as was the case in the US [26]. In 2018, the CDC reported that by May 2018, state health departments in the US received $80 \%$ of laboratory reports electronically [27].

The promotion of interoperability standards between the different types of computer systems is key to effective electronic exchange of information between different institutions. In the context of laboratory-based surveillance, this includes use of a structured message syntax, such as Health Level 7 International (HL7) and of semantic standards for coding laboratory tests and diagnostic results as well as clinical observations. A 
concerted adoption of data standards and their gradual implementation by reporting laboratories coupled with central data processing algorithms has been implemented for national laboratory-based electronic surveillance of communicable diseases in both large countries like the US $[28,29]$ and small countries like Denmark [3,11,12,30]. Universal adoption occurred faster in Denmark than in the US, where considerably more clinical laboratories had to adapt their LIMS to connect to the national reporting system $[29,30]$.

Likewise, in EU/EEA countries, the standardisation of health information systems and technologies needed for national implementation of automated M2M LIMS data reporting for communicable diseases is especially challenging in large countries with decentralised healthcare systems. This is further compounded by the different health system structures, e.g. mix of public/ private laboratory and healthcare service providers. The lack of a legal basis for automated laboratorybased surveillance in many countries makes clinical laboratory participation voluntary and requires IT services to adapt LIMS, which involves investing time and money outside their core business. As reported in this survey, the main obstacles to automated laboratorybased surveillance were insufficient IT support, lack of financial incentive followed by lack of legal mandate for automated data reporting. From the legal standpoint, it is important to integrate automated laboratory data reporting workflows with notifications by authorised health personnel for diseases or conditions under national statutory surveillance [30]. In addition, health data sharing with public health authorities must guarantee the personal data protection safeguards [31].

For two decades, the US has invested in laboratory electronic data transmission using a standard reporting process and format in collaboration with clinical laboratories, LIMS software developers and vendors, and public health agencies $[28,29]$. Equally, close partnership between all stakeholders, including clinical microbiologists, suppliers of LIMS, clinical users, public health epidemiologists and political decision-makers has been key to the success of the Danish Microbiology Database ( $\mathrm{MiBa}$ ) project, as well as its further application to monitor healthcare-associated infections and vaccine effectiveness through cross-linkage with other public registries such as administrative healthcare databases and national vaccine registries [30]. The $\mathrm{MiBa}$ receives copies of positive and negative test reports from all departments of clinical microbiology, and provides data in real-time for the surveillance of communicable diseases, thereby enabling rapid detection of outbreaks and timely analysis of trends [30,32]. After extensive validation studies for completeness and accuracy against conventional surveillance, the $\mathrm{MiBa}$ has replaced manual data reporting in Denmark for a number of diseases under surveillance, including influenza [32], pertussis [30], Clostridiodes difficile infection [12], Lyme neuroborreliosis [3] and healthcare-associated infections [11].
It is encouraging that in the present survey, $13 \mathrm{EU} / \mathrm{EEA}$ countries reported that automated laboratory data transmission was made operational for cluster detection analysis and early warning at the national level. Notably, automated reporting had replaced conventional surveillance after epidemiological validation in six countries. These findings provide further evidence of the added value of this novel surveillance approach for gains in efficiency and public health effectiveness, as also reported for the detection of hospital outbreaks [8], hepatitis A contact prophylaxis [33] and alerting of antimicrobial resistance outbreaks [7].

This study has several limitations. The self-reporting nature of the survey makes data subject to subjective interpretation by the national experts collecting the information. Possible variance in inter-reporter understanding of the survey terms was to some degree reduced by piloting the questionnaire and developing a glossary of terms and definitions via a series of discussions held individually and in the National Focal Points Forum. It was also reduced to some degree by performing a bilateral validation of each national dataset by the investigators and survey responders. As some questions, such as the use of laboratory data for early warning at the national public health level and frequency of cluster detection analysis, were only addressed to countries performing automated LIMS data reporting, we do not know to what degree this public health output differed in countries using manual data reporting. Looking to the future, the ECDC microbiology strategy envisions that in 2022, at least $90 \%$ of EU/EEA countries will be using real-time, automated $\mathrm{M}_{2} \mathrm{M}$ reporting of clinical laboratory data to national surveillance programmes [34]. Achieving this will require new policies and health system investments in many of these countries. However, such an advanced European-wide e-surveillance framework would open up further opportunity for international reporting, and the timely detection and management of cross-border health threats. The feasibility of translating national laboratory-based electronic surveillance approaches to the European surveillance level will be explored jointly by ECDC and the European Commission as part of broader EU digital health support programmes.

ECDC National Focal Points laboratory e-reporting survey group

Franz Allerberger, Austrian Agency for Health and Food Safety (AGES), Vienna, Austria

Yves Dupont, Sciensano, Brussels, Belgium

Steven Van Gucht, Sciensano, Brussels, Belgium

Sophie Quoilin, Sciensano, Brussels, Belgium

Iva Christova, National Centre of Infectious and Parasitic Diseases, Sofia, Bulgaria

Blazenka Hunjak, Croatian Institute of Public Health, Zagreb, Croatia 
Christos Karagiannis, Nicosia General Hospital, Stovolos, Nicosia, Cyprus

Pavla Krrížová, National Institute of Public Health, Prague, Czechia

Jitka Částková, National Institute of Public Health, Prague, Czechia

Eva Møller Nielsen, Statens Serum Institut, Copenhagen, Denmark

Jonas Kähler, Statens Serum Institut, Copenhagen, Denmark

Rita Peetso, Health Board, Tallinn, Estonia

Saara Salmenlinna, National Institute for Health and Welfare (THL), Helsinki, Finland

Teemu Möttönen, National Institute for Health and Welfare (THL), Helsinki, Finland

Bruno Coignard, Santé Publique France, Saint-Maurice, France

Michaela Diercke, Robert Koch Institute, Berlin, Germany

Alkiviadis Vatopoulos, National School of Public Health, Athens, Greece

Ákos Tóth, National Public Health Center, Budapest, Hungary

Karl Gustaf Kristinsson, Landspitali University Hospital, Reykjavik, Iceland

Eleanor McNamara, Public Health Laboratory, Dublin, Ireland

Annalisa Pantosi, Istituto Superiore di Sanità (ISS), Rome, Italy

Violeta Mavcutko, Centre for Disease Prevention and Control, Riga, Latvia

Algirdas Griškevičius, National Public Health Surveillance Laboratory, Vilnius, Lithuania

Joël Mossong, Laboratoire National de Santé (National Health Laboratory), Luxembourg

Christopher Barbara, Mater Dei Hospital, Msida, Malta

Titia Kortbeek, Rijksinstituut voor Volksgezondheid en Milieu (RIVM), Bilthoven, Netherlands

Dominique Caugant, Norwegian Institute of Public Health, Oslo, Norway

Ulf Dahle, Norwegian Institute of Public Health, Oslo, Norway

Line Vold, Norwegian Institute of Public Health, Oslo, Norway

Anna Skoczyńska, National Medicines Institute, Warsaw, Poland

Jorge Machado, National Institute of Health Dr Ricardo Jorge (INSA), Lisbon, Portugal

Gabriel Ionescu, ‘Cantacuzino' National Institute, Bucharest, Romania

Lucia Madarova, Regional Public Health Authority, Banska Bystrica, Slovakia
Mária Avdičová, Regional Public Health Authority, Banska Bystrica, Slovakia

Metka Paragi, National Institute of Public Health, Ljubljana, Slovenia

Julio Moreno Vazquez, Instituto de Salud Carlos III, Majadahonda, Spain

Karin Tegmark Wisell, Folkhälsomyndigheten, Solna, Sweden

Maria Zambon, Public Health England, London, United Kingdom

Acknowledgements

Bruno Ciancio and Gaetan Guyodo (ECDC) for their comments on the questionnaire.

Conflict of interest

None declared.

\section{Authors' contributions}

$\mathrm{KL}, \mathrm{LE}, \mathrm{EB}$ and $M S$ designed the study questionnaire. $\mathrm{KL}$, $\mathrm{LE}$ and $M S$ validated and analysed the data. $K L$ and LE administered the survey. $K L$ and MS drafted the manuscript. EB and LE critically reviewed the manuscript.

The following members of the ECDC National Focal Points laboratory e-reporting study team (FA, YD, SG, SQ, IC, BH, CK, PK, JC, EM, JK, RP, SS, TM, BC, MD, AV, AT, KK, EM, AP, VM, AG, JM, CB, TK, DC, UD, LV, AS, JM, GI, LM, MA, MP, JV, $K T, M Z$ ) contributed to piloting and revising the draft survey questionnaire, the data collection and the validation of the survey results.

All authors have read and approved the final manuscript.

\section{References}

1. Enki DG, Noufaily A, Garthwaite PH, Andrews NJ, Charlett A, Lane C, et al. Automated biosurveillance data from England and Wales, 1991-2011. Emerg Infect Dis. 2013;19(1):35-42. https://doi.org/10.3201/eid1901.120493 PMID: 23260848

2. Colson P, Rolain JM, Abat C, Charrel R, Fournier PE, Raoult D. EPIMIC: A Simple Homemade Computer Program for Real-Time EPIdemiological Surveillance and Alert Based on MICrobiological Data. PLoS One. 2015;10(12):e0144178. https://doi.org/10.1371/journal.pone.0144178 PMID: 26658293

3. Dessau RB, Espenhain L, Mølbak K, Krause TG, Voldstedlund $M$. Improving national surveillance of Lyme neuroborreliosis in Denmark through electronic reporting of specific antibody index testing from 2010 to 2012. Euro Surveill. 2015;20(28):21184. https://doi.org/10.2807/1560-7917. ES2015.20.28.21184 PMID: 26212143

4. Condell O, Gubbels S, Nielsen J, Espenhain L, Frimodt-Møller $\mathrm{N}$, Engberg J, et al. Corrigendum to 'Automated surveillance system for hospital-acquired urinary tract infections in Denmark' [Journal of Hospital Infection 93 (2016) 290-296]. J Hosp Infect. 2016;94(4):410. https://doi.org/10.1016/j. jhin.2016.09.001 PMID: 27665312

5. Ridgway JP, Sun X, Tabak YP, Johannes RS, Robicsek A. Performance characteristics and associated outcomes for an automated surveillance tool for bloodstream infection. Am I Infect Control. 2016;44(5):567-71. https://doi.org/10.1016/j. ajic.2015.12.044 PMID: 26899530

6. Huart M, Bedubourg G, Abat C, Colson P, Rolain JM, Chaudet $\mathrm{H}$, et al. Implementation and Initial Analysis of a Laboratory Based Weekly Biosurveillance System, Provence-Alpes-Côte d'Azur, France. Emerg Infect Dis. 2017;23(4):582-9. https://doi. org/10.3201/eid2304.161399 PMID: 28322712 
7. O'Brien TF, Clark A, Peters R, Stelling J. Why surveillance of antimicrobial resistance needs to be automated and comprehensive. J Glob Antimicrob Resist. 2019;17:8-15. https://doi.org/10.1016/j.jgar.2018.10.011 PMID: 30326273

8. Stachel A, Pinto G, Stelling J, Fulmer Y, Shopsin B, Inglima $\mathrm{K}$, et al. Implementation and evaluation of an automated surveillance system to detect hospital outbreak. Am J Infect Control. 2017;45(12):1372-7. https://doi.org/10.1016/j. ajic.2017.06.031 PMID: 28844384

9. Effler $P$, Ching-Lee $M$, Bogard A, leong MC, Nekomoto T, Jernigan D. Statewide system of electronic notifiable disease reporting from clinical laboratories: comparing automated reporting with conventional methods. JAMA. 1999;282(19):1845-50. https://doi.org/10.1001/ jama.282.19.1845 PMID: 10573276

10. Samoff E, Fangman MT, Fleischauer AT, Waller AE, Macdonald PD. Improvements in timeliness resulting from implementation of electronic laboratory reporting and an electronic disease surveillance system. Public Health Rep. 2013;128(5):393-8. https://doi.org/10.1177/003335491312800510 PMID: 23997286

11. Gubbels S, Nielsen J, Voldstedlund M, Kristensen B, Schønheyder HC, Ellermann-Eriksen S, et al. National Automated Surveillance of Hospital-Acquired Bacteremia in Denmark Using a Computer Algorithm. Infect Control Hosp Epidemiol. 2017;38(5):559-66. https://doi.org/10.1017/ ice.2017.1 PMID: 28274300

12. Chaine M, Gubbels S, Voldstedlund M, Kristensen B, Nielsen J, Andersen LP, et al. Description and validation of a new automated surveillance system for Clostridium difficile in Denmark. Epidemiol Infect. 2017;145(12):2594-602. https:// doi.org/10.1017/So950268817001315 PMID: 28689506

13. Abat C, Chaudet H, Colson P, Rolain JM, Raoult D. Real-Time Microbiology Laboratory Surveillance System to Detect Abnormal Events and Emerging Infections, Marseille, France. Emerg Infect Dis. 2015;21(8):1302-10. https://doi.org/10.3201/ eid2108.141419 PMID: 26196165

14. European Centre for Disease Prevention and Control (ECDC). EU Laboratory Capability Monitoring System (EULabCap) - Report on 2016 survey of EU/EEA country capabilities and capacities. Stockholm: ECDC; 2018. Available from: https://ecdc.europa. eu/sites/portal/files/documents/2016_EULabCap_EUreport_ web_300418_final.pdf

15. European Centre for Disease Prevention and Control (ECDC). ECDC public health microbiology strategy 2018-2022. Stockholm: ECDC; 2017. Available from: https://ecdc.europa. eu/sites/portal/files/documents/ECDC-public-healthmicrobiology-strategy-2018-2022.pdf

16. European Centre for Disease Prevention and Control (ECDC). Coordinating Competent Bodies: structures, interactions and terms of reference. Stockholm: ECDC; 7 Dec 2012 Available from: https://www.ecdc.europa.eu/sites/portal/files/media/ en/aboutus/governance/competent-bodies/Documents/ coordinating-competent-bodies-structures-terms-of-referenceand-interactions-w-Annexes.pdf

17. European Commission (EC). Commission Implementing Decision (EU) 2018/945 of 22 June 2018 on the communicable diseases and related special health issues to be covered by epidemiological surveillance as well as relevant case definitions. Brussels: Official Journal of the European Union. 6.72018:L 170. Available from: https://eur-lex.europa.eu/ legal-content/EN/TXT/PDF/ ?uri=CELEX:32018D0945\&from =EN

18. Directorate-General for Health ad Food Safety, European Commission (EC). The new EU one health action plan against antimicrobial resistance. Luxembourg: Publications Office of the European Union;19 Oct 2018. Available from: https://op.europa.eu/en/publication-detail/-/publication/ d6e681ca-d66d-11e8-9424-01aa75ed71a1

19. European Centre for Disease Prevention and Control (ECDC), European Food Safety Authority (EFSA) Panel on Biological Hazards (BIOHAZ), European Medicines Agency (EMA) Committee for Medicinal Products for Veterinary Use (CVMP). ECDC, EFSA and EMA Joint Scientific Opinion on a list of outcome indicators as regards surveillance of antimicrobial resistance and antimicrobial consumption in humans and foodproducing animals. EFSA J. 2017;15(10):e05017. PMID: 32625307

20. Technopedia. Laboratory Information Management System (LIMS). [Accessed 20 Sep 2020]. Available from: https://www.techopedia.com/definition/8085/ laboratory-information-management-system-lims

21. IoT agenda. Definition: machine-to-machine (M2M). [Accessed 20 Sep 2020]. Available from: https://internetofthingsagenda. techtarget.com/definition/machine-to-machine-M2M

22. Wurtz R, Cameron BJ. Electronic laboratory reporting for the infectious diseases physician and clinical microbiologist. Clin Infect Dis. 2005;40(11):1638-43. https://doi. org/10.1086/429904 PMID: 15889362
23. European Committee on Antimicrobial Susceptibility Testing (EUCAST). EUCAST Breakpoint tables v 8.0. Basel: European Society of Clinical Microbiology and Infectious Diseases (ECMID). 1 Jan 2018. Available from: http://www.eucast.org/ eucast_news/news_singleview/?tx_ttnews $\% 5 B t t \_n e w s \% 5 D=2$ 48\&cHash=91e3efo9a79b333746462d8854ee016d

24. Kahlmeter G. Clinical microbiology and infectious diseases working together. An ESCMID perspective. Presentation at Ulusal Klinik Mikrobiyoloji Kongresi; 2011; Antalya, Turkey. Available from: https://www.klimud.org/public/uploads/ dosya/1352555609.pdf

25. European Centre for Disease Prevention and Control (ECDC). Surveillance systems overview for 2016. Stockholm: ECDC; 4 May 2018. Available from: https://www.ecdc.europa.eu/en/ publications-data/surveillance-systems-overview-2016

26. Lamb E, Satre J, Hurd-Kundeti G, Liscek B, Hall CJ, Pinner RW, et al. Update on progress in electronic reporting of laboratory results to public health agencies - United States, 2014. MMWR Morb Mortal Wkly Rep. 2015;64(12):328-30. PMID: 25837244

27. Centers for Disease Control and Prevention (CDC). Surveillance Strategy Report - Pulse Check. Atlanta: CDC. [Accessed 20 Sep 2019]. Available from: https://www.cdc.gov/surveillance/ initiatives/pulseCheck-our-progress.html

28. Jernigan DB. Electronic laboratory-based reporting: opportunities and challenges for surveillance. Emerg Infect Dis. 2001;7(3) Suppl;538. https://doi.org/10.3201/ eido707.017717 PMID: 11485655

29. Gluskin RT, Mavinkurve M, Varma JK. Government leadership in addressing public health priorities: strides and delays in electronic laboratory reporting in the United States. Am J Public Health. 2014;104(3):e16-21. https://doi.org/10.2105/ AJPH.2013.301753 PMID: 24432922

30. Voldstedlund M, Haarh M, Mølbak K, MiBa Board of Representatives. The Danish Microbiology Database (MiBa) 2010 to 2013. Euro Surveill. 2014;19(1):20667. https://doi. org/10.2807/1560-7917.ES2014.19.1.20667 PMID: 24434175

31. European Commission (EC). Regulation (EU) $2016 / 679$ of the European Parliament and of the Council of 27 April 2016 on the protection of natural persons with regard to the processing of personal data and on the free movement of such data, and repealing Directive 95/46/EC (General Data Protection Regulation). 4.5.2016:L 119. Available from: https://eur-lex. europa.eu/eli/reg/2016/679/oj

32. Bragstad K, Emborg H, Fischer TK, Voldstedlund M, Gubbels S, Andersen $B$, et al. Low vaccine effectiveness against influenza $\mathrm{A}\left(\mathrm{H}_{3} \mathrm{~N}_{2}\right)$ virus among elderly people in Denmark in 2012/13-a rapid epidemiological and virological assessment. Euro Surveill. 2013;18(6):20397. PMID: 23410258

33. Moore KM, Reddy V, Kapell D, Balter S. Impact of electronic laboratory reporting on hepatitis A surveillance in New York City. J Public Health Manag Pract. 2008;14(5):437-41. https://doi.org/10.1097/01.PHH.0000333877.78443.fo PMID: 18708886

34. European Centre for Disease Prevention and Control (ECDC). ECDC strategic framework for the integration of molecular and genomic typing into European surveillance and multicountry outbreak investigations. Stockholm: ECDC; 4 Apr 2019. Available from: https://ecdc.europa.eu/en/publications-data/ ecdc-strategic-framework-integration-molecular-and-genomictyping-european

\section{License, supplementary material and copyright}

This is an open-access article distributed under the terms of the Creative Commons Attribution (CC BY 4.0) Licence. You may share and adapt the material, but must give appropriate credit to the source, provide a link to the licence and indicate if changes were made.

Any supplementary material referenced in the article can be found in the online version.

This article is copyright of the authors or their affiliated institutions, 2020. 